\title{
MESHLESS LARGE PLASTIC DEFORMATION ANALYSIS CONSIDERING WITH A FRICTION COEFFICIENT BY TRIPLE-RECIPROCITY BOUNDARY ELEMENT METHOD
}

\author{
YOSHIHIRO OCHIAI \\ Department of Mechanical Engineering, Kindai University, Japan.
}

\begin{abstract}
In general, internal cells are required to solve large deformation problems using a conventional boundary element method (BEM). However, in this case, the merit of BEM, which is the ease of data preparation, is lost. Triple-reciprocity BEM enables us to solve elastoplasticity problems with a small plastic deformation. In this study, it is shown that two-dimensional large plastic deformation problems with a friction coefficient can be solved without the use of internal cells, by the triple-reciprocity BEM. Initial stress and strain formulations are adopted and the initial stress or strain distribution is interpolated using boundary integral equations. In this method, only boundary elements are remeshed. A new computer program is developed and used to solve several problems.

Key words: BEM; large plastic deformation; initial stress method; numerical analysis; strain hardening; thin plate spline
\end{abstract}

\section{INTRODUCTION}

The finite element method (FEM) requires remeshing several times during large-plastic-deformation analysis. Elastoplastic problems can be solved by a conventional boundary element method (BEM) using internal cells for domain integrals [1, 2]. In this case, however, the merit of BEM, which is ease of data preparation, is lost. On the other hand, several countermeasures have been considered. Ochiai has proposed the triple-reciprocity BEM without the use of internal cells for elastoplastic problems [3]. With this method, a highly accurate solution can be obtained using only fundamental solutions of a low order and by reducing the need for data preparation. Ochiai and Kobayashi applied triple-reciprocity BEM without internal cells to two-dimensional elastoplastic problems using initial stress and strain formulations [3, 4]. However, the weak point in our previous studies was that the numerical examples are elastoplasticity problems with a small plastic deformation and too simple to substantiate the theory.

In this study, triple-reciprocity BEM is applied to large-plastic-deformation problems. The initial stress and strain formulations are adopted and the theory is expressed using a few fundamental solutions. In this method, only boundary elements are remeshed. Arbitrary distributions of the initial stress or strain for elastoplastic analysis are interpolated using boundary integral equations and internal points. This interpolation corresponds to a thin plate spline. In this method, strong singularities in the calculation of stresses at internal points become weak. A new computer program was developed and applied to several elastoplastic problems to clearly demonstrate the theory. A constrained upsetting problem can be easily solved without locking and mesh rezoning by the present method.

\section{THEORY}

\subsection{Initial stress formulation}

To analyze large-plastic-deformation problems using the initial stress formulation, the following boundary integral equation must be solved $[1,2]$. 


$$
c_{i j} \dot{u}_{j}=\int_{\Gamma}\left[u_{i j}^{[1]} \dot{p}_{j}-p_{i j} \dot{u}_{j}\right] d \Gamma+\int_{\Omega} \varepsilon_{i j k}^{[1]} \dot{\sigma}_{l j k}^{[1]} d \Omega
$$

Here, $\dot{\sigma}_{l j k}^{[1]}$ is the initial stress rate and $c_{i j}$ is the free coefficient. $u_{j}$ and $p_{j}$ are the $j$ th component of the displacement rate and surface traction rate, respectively. Upper notation [1] is a conventional term. $\Gamma$ and $\Omega$ are the boundary and domain, respectively. As shown in eqn (1), when there is an arbitrary initial stress rate, a domain integral becomes necessary. Denoting the distance between the observation point and the loading point as $r$, Kelvin's solution $u_{i j}^{[1]}$ and $p_{i j}$ are given by

$$
\begin{gathered}
u_{i j}^{[1]}=\frac{1}{8 \pi(1-v) G}\left[(3-4 v) \delta_{i j} \ln \left(\frac{1}{r}\right)+r,{ }_{i} r,{ }_{j}\right] \\
p_{i j}=\frac{-1}{4 \pi(1-v) r}\left\{\left[(1-2 v) \delta_{i j}+2 r,_{i} r,{ }_{j}\right] \frac{\partial r}{\partial n}-(1-2 v)\left(r,{ }_{i} n_{j}-r,{ }_{j} n_{i}\right)\right\},
\end{gathered}
$$

where $v$ is Poisson's ratio and $G$ is the shear modulus. The $i$ th component of a unit normal vector is denoted by $n_{i}$. Moreover, let us set $v^{\prime}=v /(1+v)$ for the plane stress and $r_{i}=f r / f x_{i}$. For large deformation Poisson's ratio $v$ approximately 0.5 is assumed. The function $\varepsilon_{i j k}^{[1]}$ in eqn (1) is given by [1]

$$
\varepsilon_{i j k}^{[1]}=\left[(1-2 v)\left(\delta_{i j} r,_{k}+\delta_{i k} r,{ }_{j}\right)-\delta_{j k} r,_{i}+2 r,{ }_{i} r,{ }_{j} r{ }_{k}\right] \frac{-1}{8 \pi(1-v) G r} .
$$

\subsection{Interpolation of initial stress}

Interpolation using boundary integrals is necessary to avoid the domain integral in eqn (1). The distribution of the initial stress $\dot{\sigma}_{l j k}^{[1]}$ in the case of a two-dimensional problem is interpolated using the integral equation to transform the domain integral into a boundary integral. The following equations are used for interpolation [5-10]:

$$
\begin{gathered}
\nabla^{2} \dot{\sigma}_{l j k}^{[1] S}=-\dot{\sigma}_{l j k}^{[2] S}, \\
\nabla^{2} \dot{\sigma}_{l j k}^{[2] S}=-\sum_{m=1}^{M} \dot{\sigma}_{l j k(m)}^{[3] P},
\end{gathered}
$$

where $\nabla^{2}=\partial^{2} / \partial x^{2}+\partial^{2} / \partial y^{2}$. From eqns (5) and (6), we obtain

$$
\nabla^{4} \dot{\sigma}_{l j k}^{[1] S}=\sum_{m=1}^{M} \dot{\sigma}_{I j k(m)}^{[3] P} .
$$

This equation is the same type of equation as for the displacement $\left(\dot{\sigma}_{I j k}^{[1]}\right)$ of a fictitious thin plate with point loads $\left(\dot{\sigma}_{l j k}^{[3] P}\right)$, as shown in Fig. 1 . The $\dot{\sigma}_{i j k}^{[1]}$ values at several points and on the boundary are given, and the unknown point load $P$ is obtained inversely [4, 7]. The term $\dot{\sigma}_{l j k}^{[2] S}$ corresponds to the sum of the curvatures of $\dot{\sigma}_{l j k}^{[1]}$. This fictitious thin plate is simply supported, and the $\dot{\sigma}_{l j k}^{[2] S}$ values on the boundary are zero. We emphasize that eqns (5) and (6) can be used to interpolate the complicated distribution of the initial stress $\dot{\sigma}_{l j k}^{[1]}$. These equations are the same as those used to generate a free-form surface using an integral equation $[6,7]$. In this study, the distribution of the initial stress is assumed to be represented by a 2.5 -dimensional free-form surface. In this method, each component of the initial stress $\dot{\sigma}_{l j k}^{[1]}(j, k=1,2)$ is interpolated. 


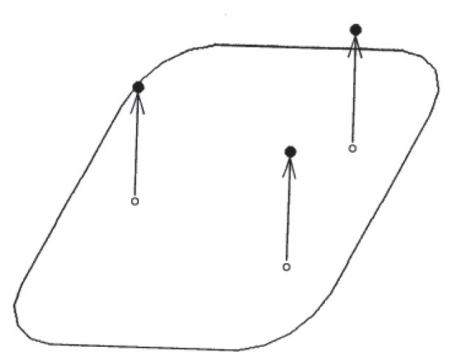

(a) Given internal points and boundary

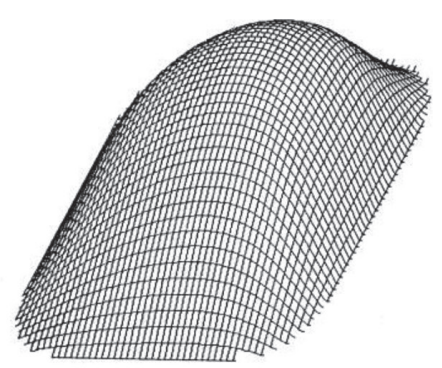

(b) Obtained surface $\dot{\sigma}_{I j k}^{[1]}$

Figure 1: Fictitious thin plate with unknown point loads used for interpolation.

\subsection{Representation of initial stress by integral equation}

The distribution of the initial stress is represented by an integral equation. The harmonic function $T^{[1]}$ and biharmonic function $T^{[2]}$ are generally given by

$$
\begin{aligned}
T^{[1]} & =\frac{1}{2 \pi}\left[\ln \left(\frac{1}{r}\right)+B\right] . \\
T^{[2]} & =\frac{r^{2}}{8 \pi}\left[\ln \left(\frac{1}{r}\right)+B+1\right],
\end{aligned}
$$

where $B$ is an arbitrary constant. Denoting the number of points $\dot{\sigma}_{l j k}^{[3] P}$ as $M$, the curvature of the initial stress rate $\dot{\sigma}_{l j k}^{[2] S}$ is given by Green's second identity and eqn (8) as [4-7, 11-13]

$$
c \dot{\sigma}_{l j k}^{[2] S}(P)=\int_{\Gamma}\left\{T^{[1]}(P, Q) \frac{\partial \dot{\sigma}_{l j k}^{[2] S}(Q)}{\partial n}-\frac{\partial T^{[1]}(P, Q)}{\partial n} \dot{\sigma}_{l j k}^{[2] S}(Q)\right\} d \Gamma+\sum_{m=1}^{M} T^{[1]} \dot{\sigma}_{l j k(m)}^{[3] P} .
$$

The initial stress rate $\dot{\sigma}_{l j k}^{[1]}$ is given by Green's theorem and eqns (7) and (8) as [4-7]

$$
\begin{gathered}
c \dot{\sigma}_{l j k}^{[1] S}(P)=-\sum_{f=1}^{2}(-1)^{f} \int_{\Gamma}\left\{T^{[f]}(P, Q) \frac{\partial \dot{\sigma}_{l j k}^{[f] S}(Q)}{\partial n}-\frac{\partial T^{[f]}(P, Q)}{\partial n} \dot{\sigma}_{l j k}^{[f] S}(Q)\right\} d \Gamma \\
-\sum_{m=1}^{M} T^{[2]} \dot{\sigma}_{l j k(m)}^{[3] P}
\end{gathered}
$$

where $c=0.5$ on the smooth boundary and $c=1$ in the domain. $\dot{\sigma}_{l i k}^{[2] S}(\mathrm{Q})$ is zero because the fictitious thin plate is simply supported. $\partial \dot{\sigma}_{l j k}^{[2] S} / \partial n$ is zero for symmetric boundary. From eqns (10) and (11),

$$
\begin{gathered}
c \dot{\sigma}_{l j k}^{[1] S}(P)=\int_{\Gamma}\left\{T^{[1]}(P, Q) \frac{\partial \dot{\sigma}_{l j k}^{[1] S}(Q)}{\partial n}-\frac{\partial T^{[1]}(P, Q)}{\partial n} \dot{\sigma}_{l j k}^{[1] S}(Q)\right\} d \Gamma \\
-\int_{\Gamma}\left\{T^{[2]}(P, Q) \frac{\partial \dot{\sigma}_{l j k}^{[2] S}(Q)}{\partial n} d \Gamma-\sum_{m=1}^{M} T^{[2]}(P, q) \dot{\sigma}_{l j k(m)}^{[3] P}(q),\right. \\
0=\int_{\Gamma} T^{[1]}(P, Q) \frac{\partial \dot{\sigma}_{l j k}^{[2] S}(Q)}{\partial n} d \Gamma+\sum_{m=1}^{M} T^{[1]}(P, q) \dot{\sigma}_{l j k(m)}^{[3] P}(q) .
\end{gathered}
$$


On the other hand, $\partial \dot{\sigma}_{l j k}^{[2] S} / \partial n$ can be assumed to be zero on the symmetric boundary. For internal points, the following equation is obtained:

$$
\begin{gathered}
c \dot{\sigma}_{l j k}^{[1] S}(p)=\int_{\Gamma}\left\{T^{[1]}(p, Q) \frac{\partial \dot{\sigma}_{l j k}^{[1] S}(Q)}{\partial n}-\frac{\partial T^{[1]}(p, Q)}{\partial n} \dot{\sigma}_{l j k}^{[1] S}(Q)\right\} d \Gamma \\
\left.-\int_{\Gamma} \frac{\partial T^{[2]}(p, Q)}{\partial n} \dot{\sigma}_{l j k}^{[2] S}(Q)\right\} d \Gamma-\sum_{m=1}^{M} T^{[2]}(p, q) \dot{\sigma}_{l j k(m)}^{[3] P}(q) .
\end{gathered}
$$

In practice, unknown $\partial \dot{\sigma}_{l j k}^{[1] S}(Q) / \partial n, \partial \dot{\sigma}_{l j k}^{[2] S}(Q) / \partial n$ and $\dot{\sigma}_{l j k(m)}^{[3] P}$ must be obtained from $\dot{\sigma}_{l j k}^{[1]}(P)$ and $\dot{\sigma}_{l j k}^{[1]}(p)$. Replacing $\dot{\sigma}_{l j k}^{[f] S}(Q), \partial \dot{\sigma}_{l j k}^{[f] S}(Q) / \partial n$ and $\dot{\sigma}_{l j k(m)}^{[3] P}$ with vectors $\mathrm{E}^{[\mathrm{f}]}, \mathrm{V}^{[\mathrm{f}]}$ and $\mathrm{E}^{[3] \mathrm{P}}(\mathrm{q})$, respectively, we obtain the following equations from eqns (12)-(14) [4].

$$
\begin{gathered}
\mathrm{E}^{[1]}(\mathrm{P})=\mathrm{G}_{1} \mathrm{~V}^{[1]}-\mathrm{H}_{1} \mathrm{E}^{[1]}(\mathrm{P})-\mathrm{G}_{2} \mathrm{~V}^{[2]}-\mathrm{G}_{1}{ }^{\mathrm{P}} \mathrm{E}^{[3] \mathrm{P}}(\mathrm{q}) \\
0=\mathrm{G}_{1} \mathrm{~V}^{[1]}+\mathrm{G}_{1}{ }^{\mathrm{P}} \mathrm{E}^{[3] \mathrm{P}}(\mathrm{q}) \\
\mathrm{E}^{[1]}(\mathrm{p})=\mathrm{G}_{3} \mathrm{~V}^{[1]}-\mathrm{H}_{3} \mathrm{E}^{[1]}(\mathrm{P})-\mathrm{G}_{4} \mathrm{~V}^{[2]}-\mathrm{G}_{4}{ }^{\mathrm{P}} \mathrm{E}^{[3] \mathrm{P}}(\mathrm{q})
\end{gathered}
$$

The details of this procedure are given in reference [4]. From eqns (15)-(17),

$$
\left[\begin{array}{c}
\mathbf{V}^{[1]} \\
\mathbf{V}^{[2]} \\
\mathbf{E}^{[3] P}(q)
\end{array}\right]=\mathbf{A}^{-1}\left[\begin{array}{c}
\mathbf{H}_{1} \mathbf{E}^{[1]}(P) \\
\mathbf{0} \\
\mathbf{H}_{3} \mathbf{E}^{[1]}+\mathbf{G}_{3} \mathbf{E}^{[1]}(\mathrm{p})
\end{array}\right]
$$

where $\mathbf{A}^{-1}$ is the inverse matrix. If the boundary is divided into $N_{0}$ constant elements, and $N_{1}$ internal points are used, the simultaneous linear algebraic equations with $\left(2 N_{0}+N_{1}\right)$ as unknowns must be solved.

\subsection{Triple-reciprocity BEM}

The function $\varepsilon_{i j k}^{[f]}$ is defined as

$$
\nabla^{2} \varepsilon_{i j k}^{[f+1]}=\varepsilon_{i j k}^{[f]} .
$$

Using eqns (5), (6), (18), (19) and Green's second identity, eqn (1) becomes

$$
\begin{aligned}
c_{i j} \dot{u}_{j} & =\int_{\Gamma}\left[u_{i j}^{[1]} \dot{p}_{j}-p_{i j} \dot{u}_{j}\right] d \Gamma \\
& -\sum_{f=1}^{2}(-1)^{f} \int_{\Gamma}\left\{\frac{\partial \varepsilon_{i j k}^{[f+1]}}{\partial n} \dot{\sigma}_{l j k}^{[f] S}-\varepsilon_{i j k}^{[f+1]} \frac{\partial \dot{\sigma}_{l j k}^{[f] S}}{\partial n}\right\} d \Gamma+\sum_{m=1}^{M} \varepsilon_{i j k}^{[3]} \dot{\sigma}_{l j k(m)}^{[3] P} .
\end{aligned}
$$

Next, the function $\varepsilon_{i j k}^{[f]}$ is obtained, and the relationship between the biharmonic function $A^{[1]}$ and Kelvin's solution $u_{i j}^{[1]}$ is given by

$$
u_{i j}^{[1]}=\frac{-1}{2(1-v) G} A,_{i j}^{[1]}+\frac{\delta_{i j} A_{, k k}^{[1]}}{G} .
$$


The biharmonic function $A^{[1]}$ is given as

where

$$
A^{[1]}=\frac{r^{2}}{8 \pi}\left[\ln \left(\frac{1}{r}\right)+C_{q}\right]
$$

$$
C_{q}=\frac{7-8 v}{2(3-4 v)}
$$

The displacement-strain relationship is given by

$$
\varepsilon_{i j k}^{[1]}=\frac{1}{2}\left(u_{j i, k}^{[1]}+u_{k i, j}^{[1]}\right),
$$

Next, the function $A^{[f]}$ that satisfies

$$
\nabla^{2} A^{[f+1]}=A^{[f]}
$$

is considered. $A^{[f]}$ is generally given by

$$
A^{[f]}=\frac{r^{2 f}}{2 \pi[(2 f) ! !]^{2}}\left[\ln \left(\frac{1}{r}\right)+C_{q}+\operatorname{sgn}(f-1) \sum_{e=2}^{f} \frac{1}{e}\right] .
$$

Accordingly, from eqns (24), (26), (29) and (30), $\varepsilon_{i j k}^{[f]}$ is obtained as [4]

$$
\begin{gathered}
\varepsilon_{i j k}^{[f]}=\frac{2 r^{2 f-3}}{2 \pi G\{(2 f) ! !\}^{2}}\left\langle\frac{2}{(1-v)}\left[2 f(f-1)(f-2) F_{2}+\left(3 f^{2}-6 f+2\right)\right] r,_{i} r,_{j} r_{,_{k}}\right. \\
+2\left[2 f^{2}(f-1) F_{2}+f(3 f-2)\right]\left(\delta_{i k} r,_{j}+\delta_{i j} r_{,_{k}}\right) \\
\left.+\left[2 f(f-1) F_{2}+(2 f-1)\right]\left(\delta_{i j} r,_{k}+\delta_{k i} r,_{j}+\delta_{j k} r r_{i}\right)\right\rangle,
\end{gathered}
$$

where

$$
F_{2}=\ln (r)+C_{q}-\sum_{e=2}^{f} \frac{1}{e}
$$

\subsection{Internal stresses}

The stress-strain relationship is given by

$$
\sigma_{i j k}^{[1]}=\lambda \delta_{j k} u_{m i, m}^{[1]}+\mu\left(u_{j i, k}^{[1]}+u_{k i, j}^{[1]}\right),
$$

where Lame's constant $\lambda$ and $\mu$ are defined as

$$
\begin{gathered}
\lambda=\frac{2 v G}{1-2 v} \\
\mu=\mathrm{G} .
\end{gathered}
$$

The internal stress is given by

$$
\dot{\sigma}_{i j}=\int_{\Gamma}\left[-\sigma_{k i j}^{[1]} \dot{p}_{k}-S_{k i j} \dot{u}_{k}\right] d \Gamma+\int_{\Omega} \varepsilon_{i j k s}^{[1]} \dot{\sigma}_{k s}^{[1]} d \Omega-\dot{\sigma}_{l i j}^{[1]},
$$


where $\dot{\sigma}_{I j}^{[1]}$ is the initial stress obtained from the initial strain. The functions $S_{k i j}$ and $\sigma_{i j k s}^{H[1]}$ in eqn (32) are given by

$$
\begin{aligned}
& S_{k i j}=\frac{G}{2 \pi(1-v) r^{2}}\left[2 \frac{\partial r}{\partial n}\left\{(1-2 v) \delta_{i j} r_{, k}+v\left(\delta_{i k} r_{, j}+\delta_{j k} r_{, i}\right)-4 r_{, i} r_{, j} r_{, k}\right\}\right. \\
& \left.+2 v\left(n_{i} r_{, j} r_{, k}+n_{j} r_{i} r_{, k}\right)+(1-2 v)\left(2 n_{k} r_{, i} r_{, j}+n_{j} \delta_{i k}+n_{i} \delta_{j k}\right)-(1-4 v) n_{k} \delta_{i j}\right] \\
& \varepsilon_{i j k s}^{[1]}=\frac{1}{4 \pi(1-v) r^{2}}\left[2(1-2 v)\left(\delta_{i k} r,{ }_{j} r,{ }_{s}+\delta_{j k} r,{ }_{i} r,{ }_{s}-\delta_{i j} \delta_{k s}+2 \delta_{i j} r{ }_{k} r,{ }_{s}\right)\right.
\end{aligned}
$$

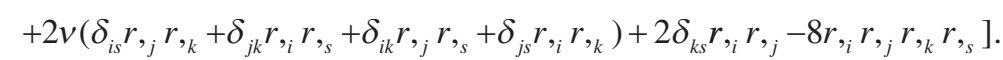

The function $\sigma_{i j k s}^{H[f]}$ is defined as

$$
\nabla^{2} \varepsilon_{i j k s}^{[f+1]}=\varepsilon_{i j k s}^{[f]}
$$

Using eqn (35) and Green's theory, eqn (32) becomes

$$
\begin{aligned}
\dot{\sigma}_{i j} & =\int_{\Gamma}\left[-\sigma_{k i j}^{[1]} \dot{p}_{k}-S_{k i j} \dot{u}_{k}\right] d \Gamma-\sum_{f=1}^{2}(-1)^{f} \int_{\Gamma}\left[\frac{\partial \varepsilon_{i j k s}^{[f+1]}}{\partial n} \dot{\sigma}_{k s}^{[f] S}-\varepsilon_{i j k s}^{[f+1]} \frac{\partial \dot{\sigma}_{k s}^{[f] S}}{\partial n}\right] d \Gamma \\
& +\sum_{m=1}^{M} \varepsilon_{i j k s}^{[3]}(p, q) \dot{\sigma}_{k s(m)}^{[3] P}-\dot{\sigma}_{l i j}^{[1]} .
\end{aligned}
$$

Using eqn (35) and the relationship between displacement and strain, $\varepsilon_{i j k s}^{[f]}$ is obtained as

$$
\begin{aligned}
& \varepsilon_{i j k s}^{[f]}(p, q)=\frac{r^{2 f-4}}{\pi(1-v)[(2 f) ! !]^{2}}\left\langle\left\{-2\left[8 f^{3} v-3 f^{2}(1+6 v)+2 f(3+4 v)-2\right]\right.\right. \\
& \left.-4 f(f-1)(f-2)(2 f v-1) F_{2}\right\} \delta_{i s} r,{ }_{j} r{ }_{k} \\
& -\left[6 f^{2} v-2 f(1+2 v)+1+2 f(f-1)(2 f v-1) F_{2}\right] \delta_{i s} \delta_{j k} \\
& +\left\{8(2 f-3)[1+f(f-3)]+f(f-1)(f-2)(f-3) F_{2}\right\} r,{ }_{i} r,{ }_{j} r,{ }_{k} r, \\
& +2\left[3 f^{2}-6 f+2+2 f(f-1)(f-2) F_{2}\right] \delta_{j k} r{ }_{i} r, \\
& -2\left\{4 f^{3}(1-v)-3 f^{2}(4-3 v)+2 f(5-2 v)-2\right. \\
& \left.-2 f(f-1)(f-2)[1-f(1-v)] F_{2}\right\}\left(\delta_{i j} r{ }_{, k} r,_{s}+\delta_{i k} r{ }_{j} r,_{s}+\delta_{s j} r_{, k} r,_{i}+\delta_{s k} r{ }_{j} r,_{i}\right) \\
& \left.-\left\{1+6 f^{2}(1-v)-2 f(3-2 v)+2 f(f-1)[2 f(1-v)-1] F_{2}\right\}\left(\delta_{i j} \delta_{k s}+\delta_{i k} \delta_{j s}\right)\right\rangle .
\end{aligned}
$$

\subsection{Initial stress}

An iterative process is used for elastoplastic analysis. The equivalent plastic strain increment is denoted as $d \varepsilon^{P}{ }_{e}$. The von Mises yield criterion is considered. The deviatoric stress tensor $S_{i j}$ is obtained from the stress rate in eqn (36). The plastic strain increments $d \varepsilon^{P}{ }_{i j}$ are determined using the Prandtl-Reuss equation

$$
d \varepsilon_{i j}^{P}=S_{i j} d \lambda
$$


where $d \lambda$ is a proportionality factor. Using this equation, the provisional plastic strain increments $d \varepsilon^{P}{ }_{i j}$ are calculated. Using the initial stress rate on the boundary and at internal points, the initial stress rate is interpolated using eqn (18). The displacement and traction rates are obtained from eqn (20) as

$$
\mathrm{HU}=\mathrm{GP}+\mathrm{D}_{1} \mathrm{E}^{[1]}(\mathrm{Q})-\mathrm{D}_{2} \mathrm{~V}^{[1]}+\mathrm{D}_{3} \mathrm{~V}^{[2]}+\mathrm{D}_{4} \mathrm{E}^{[3] \mathrm{P}}(\mathrm{q})
$$

The stress rate is obtained from eqn (36) as

$$
\sigma=\mathrm{G}^{\mathrm{A}} \mathrm{P}-\mathrm{H}^{\mathrm{A}} \mathrm{U}+\mathrm{D}_{1}^{\mathrm{A}} \mathrm{E}^{[1]}(\mathrm{Q})-\mathrm{D}_{2}^{\mathrm{A}} \mathrm{V}^{[1]}+\mathrm{D}_{3}^{\mathrm{A}} \mathrm{V}^{[2]}+\mathrm{D}_{4}^{\mathrm{A}} \mathrm{E}^{[3] \mathrm{P}}(\mathrm{q})-\sigma_{\mathrm{I}}
$$

The details of this procedure are the same as those of the procedure given in Ref. [1], with the exception of the interpolation. In this theory, the surface stress rate must be calculated to obtain the initial strain on the boundary. This surface stress rate can be obtained by the differentiation of node displacements. The initial stress rate converges using the above iterative process. New coordinates of boundary elements and internal points are calculated. If a length of the new boundary element is larger than the standard length $L_{0}$, the boundary element is divided into two boundary elements. If the distance between a boundary element and an internal point is smaller than the standard length $R_{0}$, the internal point is deleted to prevent a numerical error in the internal stress. Subsequently, the next load increment (displacement increment) is added. Coulomb's friction coefficient is considered on the contact surfaces.

\section{NUMERICAL EXAMPLE}

The large plastic deformation problem of a plate (length $20 \mathrm{~mm}$ ) subjected to tension, as shown in Fig. 2, is solved using the von Mises yield criterion. The number of discretized boundary elements is 400 and the number of internal points used for interpolation is 133, as shown in Fig. 2. Young's modulus E of $210 \mathrm{GPa}$, Poisson's ratio $v$ of 0.3 and yield stress $\sigma_{0}$ of $200 \mathrm{MPa}$ are assumed. For large deformation, Poisson's ratio $v$ of 0.48 is assumed. It is assumed that the number of time steps for the tension $(10 \mathrm{~mm})$ and the substeps for convergence are 100 and 50, respectively. Displacement load curves are shown in Fig. 3 with theoretical values for work hardening values $\mathrm{H}=0$ and $840 \mathrm{MPa}$.

The elastoplastic problem of a plane strain punch is next solved using the von Mises yield criterion. This example consists of a rigid flat punch indented into a solid plane strain

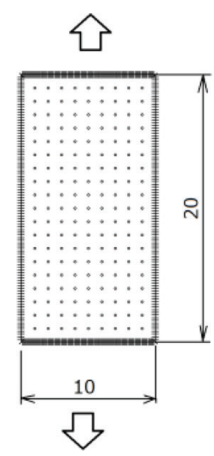

Figure 2: Tension of plate.

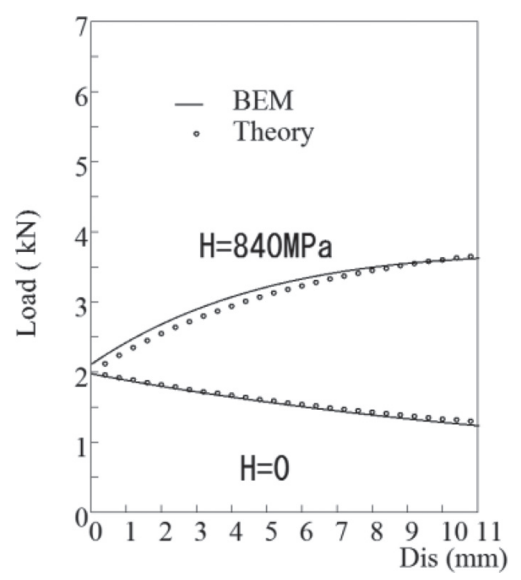

Figure 3: History of load. 
specimen, as shown in Fig. 4. First, it is assumed that there is no friction between the specimen and the punch. One-quarter of the region is used for calculation. The number of discretized boundary elements is 400 , and the number of internal points for interpolation is 153 , as shown in Fig. 5. Young's modulus E of $210 \mathrm{GPa}$, Poisson's ratio $v$ of 0.3 , and yield stress $\sigma_{0}$ of $200 \mathrm{MPa}$ are assumed. It is assumed that the number of time steps for compression ( $5 \mathrm{~mm})$ and substeps for convergence are 100 and 50, respectively. Displacement load curves without friction are shown in Fig. 6 with theoretical values for work hardening values $\mathrm{H}$ of 0 and $420 \mathrm{MPa}$. Second, the distributions of equivalent plastic strain and stress considering the friction coefficient $(\mu=0.1)$ are shown in Figs 7 and 8, respectively.

Finally, the constrained upsetting problem of a plane strain punch in Fig. 9 is solved using the von Mises yield criterion. It is assumed that there is no slip between the specimen and the punch. One-quarter of the region is used for calculation. The number of discretized boundary elements is 400 and the number of internal points used for interpolation is 153, as shown in Fig. 5. Young's modulus E of $210 \mathrm{GPa}$, Poisson's ratio $v$ of 0.3 , and yield stress $\sigma_{0}$ of $200 \mathrm{MPa}$ are assumed. It is assumed that the number of time steps for compression $(5 \mathrm{~mm})$ and substeps for convergence are 100 and 50, respectively. A work hardening value $\mathrm{H}$ of $420 \mathrm{MPa}$ is assumed. Distributions of equivalent plastic strain and stress are shown in Figs 10 and 11, respectively.

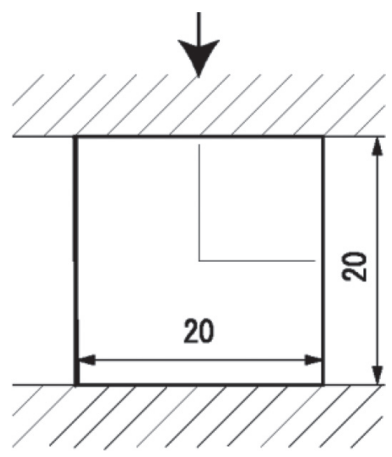

Figure 4: Plane strain punch problem.

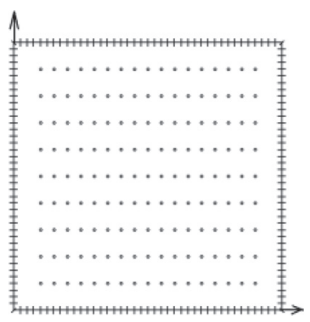

Figure. 5: Boundary elements and internal points in quarter region (Number of boundary elements: 100).

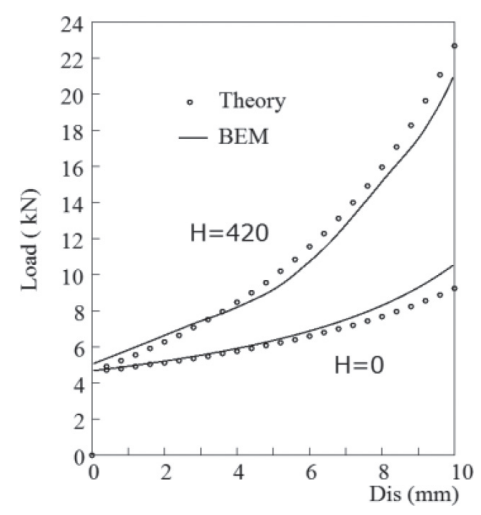

Figure 6: History of load $(\mu=0)$. 

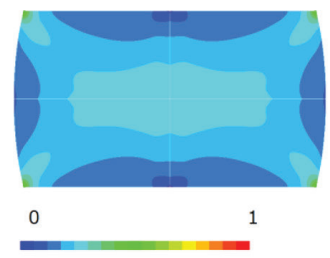

(a) $Z=5 \mathrm{~mm}$

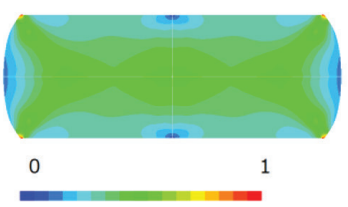

(b) $Z=8 \mathrm{~mm}$

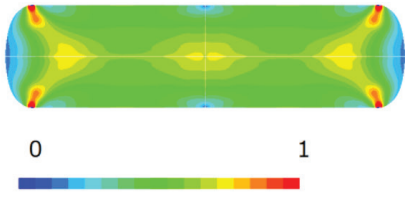

(c) $Z=10 \mathrm{~mm}$

Figure 7: Distribution of plastic strain $(\mu=0.1)$.

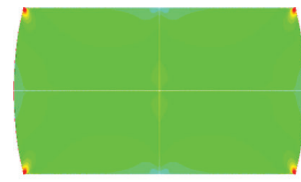

0

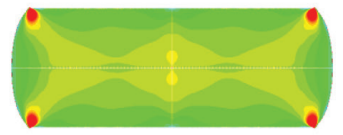

700

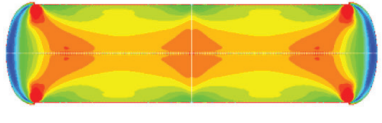

(b) $\mathrm{Z}=8 \mathrm{~mm}$

0

(b) $Z=8 \mathrm{~mm}$ (c) $Z=10 \mathrm{~mm}$

0

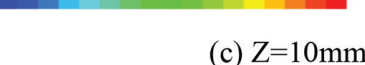

Figure 8: Equivalent stress distribution $(\mu=0.1, \mathrm{MPa})$.

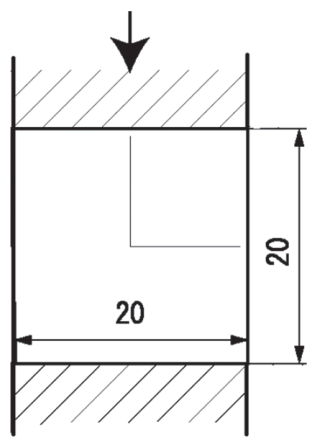

Figure 9: Constrained upsetting problem.
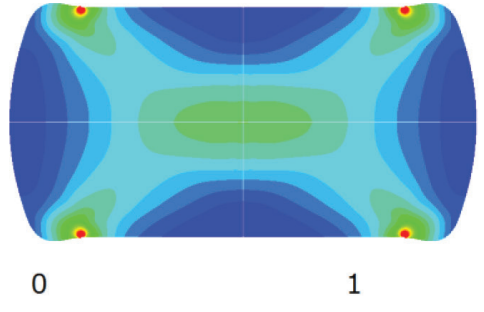

$\mathrm{Z}=5 \mathrm{~mm}$

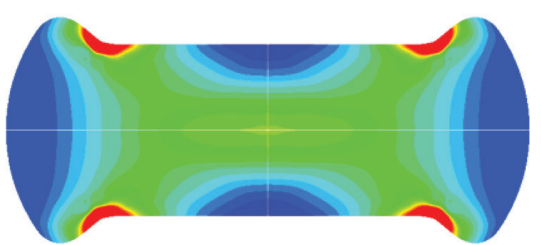

1

$$
\mathrm{Z}=10 \mathrm{~mm}
$$

Figure 10: Distribution of equivalent strain in constrained upsetting problem. 

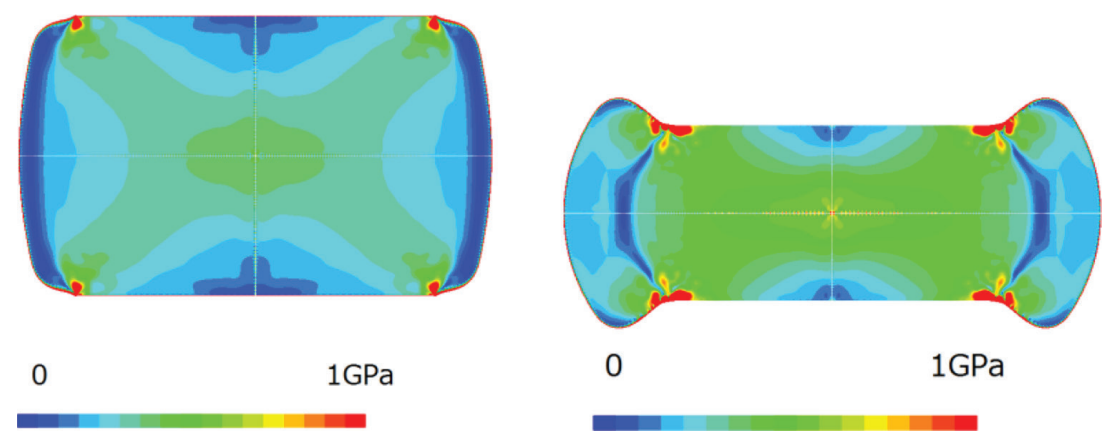

Figure 11: Equivalent stress distribution in constrained upsetting problem.

\section{CONCLUSION}

It was shown that two-dimensional large plastic deformation analysis can be carried out without the use of internal cells, by the triple-reciprocity BEM. The fundamental solutions for this analysis were shown. In this method, the strong singularity that appears in the calculation of internal stress by the ordinary BEM becomes weak. With the use of numerical examples, the effectiveness and accuracy of this method were demonstrated. In this method, the merit of BEM, which is the ease of data preparation, is not lost because internal cells are not necessary. If FEM is used, remeshing (rezoning) is necessary for large-plastic-deformation analysis. Only boundary elements are remeshed in this triple-reciprocity BEM.

\section{REFERENCES}

[1] Telles, J.C.F., The boundary element method applied to inelastic problems, SpringerVerlag, Berlin, 1983.

[2] Brebbia, C.A., Telles, J.C.F. \& Wrobel, L.C., Boundary element techniques-theory and applications in engineering, Springer-Verlag, Berlin, pp. 252-266, 1984.

[3] Ochiai, Y. \& Kobayashi, T., Initial stress formulation for elastoplastic analysis by improved multiple-reciprocity boundary element method. Engineering Analysis with Boundary Elements, 23, pp. 167-173, 1999. https://doi.org/10.1016/s0955-7997(98)00066-6

[4] Ochiai, Y. \& Kobayashi, T., Initial strain formulation without internal cells for elastoplastic analysis by triple-reciprocity BEM. International Journal for Numerical Method in Engineering, 50, pp. 1877-1892, 2001. https://doi.org/10.1002/nme.100

[5] Ochiai, Y., Three-dimensional elastoplastic analysis by triple-reciprocity boundary element method. Communications in Numerical Methods in Engineering, 23, pp. 721-732, 2007. https://doi.org/10.1002/cnm.922

[6] Ochiai, Y. \& Sekiya, T., Generation of free-form surface in CAD for dies. Advances in Engineering Software, 22, pp. 113-118, 1995. https://doi.org/10.1016/0965-9978(94)00030-m

[7] Ochiai, Y. \& Yasutomi, Z., Improved method generating a free-form surface using integral equations. Computer-Aided Geometric Design, 17(3), pp. 233-245, 2000. https://doi.org/10.1016/s0167-8396(99)00047-3 
[8] Ochiai, Y., Nishitani, H. \& Sekiya, T., Stress analysis with arbitrary body force by boundary element method. Engineering Analysis with Boundary Elements, 17, pp. 295-302, 1996. https://doi.org/10.1016/s0955-7997(96)00031-8

[9] Ochiai, Y., Multidimensional numerical integration for meshless BEM. Engineering Analysis with Boundary Elements, 27(3), pp. 241-249, 2003. https://doi.org/10.1016/s0955-7997(02)00112-1

[10] Ochiai, Y. \& Sladek, V., Numerical treatment of domain integrals without internal cells in three-dimensional BIEM formulations. CMES (Computer Modeling in Engineering \& Sciences), 6(6), pp. 525-536, 2004.

[11] Ochiai, Y., Meshless unsteady thermo-elastoplastic analysis by triple-reciprocity boundary element method. CMES (Computer Modeling in Engineering \& Sciences), 79(2), pp. 83-101, 2011. https://doi.org/10.3970/cmes.2011.079.083

[12] Ochiai, Y., Three-dimensional thermo-elastoplastic analysis by triplereciprocity boundary element method. Engineering Analysis with Boundary Elements, 35(3), pp. 478-488, 2011. https://doi.org/10.1016/j.enganabound.2010.08.018

[13] Ochiai, Y., Large plastic deformation analysis without use of internal cells by triplereciprocity BEM, Proceedings of 6th International Conference on Boundary Element Techniques, p. 293, 2005. 